\title{
The PICASSO Dark Matter Search Project
}

\author{
A. Davour for the PICASSO collaboration \\ Queen's University \\ E-mail: adavour@owl.phy.queensu.ca
}

PICASSO is an array of bubble detectors constructed to search for spin dependent interactions of weakly interacting massive particles. The bubble detectors are similar to those used in neutron dosimetry: superheated droplets of fluorocarbon $\left(\mathrm{C}_{4} \mathrm{~F}_{10}\right)$ are suspended in a polymerized gel, where a nucleus recoiling from the elastic scattering of a WIMP triggers the boiling of the superheated liquid. The explosive expansion of a droplet to a bubble will induce an acoustic wave in the gel which is picked up with piezoelectric sensors. The PICASSO collaboration has developed large volume bubble detectors of 4.5 litres. The performance of these detectors has been evaluated with several methods. The detectors are installed at SNOLAB, located at a depth of 2070 meters (5890 m.w.e.) in the Creighton mine in Sudbury. The completed setup contains 32 detectors with a combined active mass of $2.6 \mathrm{~kg}$. New results are presented, describing a promising method to distinguish alpha events from nuclear recoil events and thereby reduce the background.

Identification of dark matter 2008

August 18-22, 2008

Stockholm, Sweden 


\section{The PICASSO project}

With the compelling evidence we now have for the existence of dark matter, there are many efforts going on with the goal to discover dark matter particles. These are likely to be weakly interacting massive particles (WIMPs), and the most popular WIMP candidate is the lightest particle appearing in supersymmetric extensions to the standard model. PICASSO (Project In CAnada to Search for Supersymmetric Objects) [3], like other direct dark matter searches, has the objective to detect the nuclear recoil induced by a WIMP interacting in the detector active volume.

PICASSO is modular, consisting of a number of bubble detectors. These are similar in operation principle to those used in personal neutron dosimetry [6]. The active material is a superheated liquid (in this case $\mathrm{C}_{4} \mathrm{~F}_{10}$ ) in the form of droplets suspended in a gel matrix. The gel forms smooth walls around the droplets, without any imperfections that can act as nucleation centres. Each droplet acts as an independent bubble chamber. If a sufficient heat spike is deposited within a critical length in the superheated liquid, the droplet will undergo a rapid phase transition.

The expansion of the droplet to a bubble with a volume two orders of magnitude larger will induce a pressure wave in the gel which can be picked up by piezoelectric sensors attached to the detector walls.

From the operating principle it's clear that these detectors are threshold counters. The threshold energy required to induce the phase transition depends on the degree of superheat of the active liquid, and therefore on the temperature and pressure. At low temperatures, and low degree of superheat, only the most energetic recoils will be detected, while at higher temperatures also smaller deposited energies will be visible (see figure 1). Closer to fifty degrees even interactions of gammas and minimum ionizing particles will be detectable. By scanning over temperatures (at constant pressure) the response characteristic for different interactions can be evaluated and we get a good understanding of the detector properties and our backgrounds. It's useful to understand that each temperature corresponds to a threshold energy as demonstrated in figure 1, and that in this way the temperature scale can be converted to an energy scale.

The attractive feature of the response behaviour is that there is a temperature region where the detectors are sensitive to WIMP interactions but insensitive to gammas or muons.

The most serious background, limiting the signal sensitivity, is that from internal contamination of alpha-active elements. There is no shielding against internal contamination, and the detectors are sensitive to alphas in the whole signal temperature region. A lot of effort is going into reducing this background by purification of the ingredients. One problem is the heavy salt added to the gel in order to match the density of the $\mathrm{C}_{4} \mathrm{~F}_{10}$, which carries with it traces of radioactive elements. A new formula of the gel has been tried out, which eliminates the need for the salt.

Since each droplet is an independent detection volume, the detector is continuously active. When too many bubbles have formed, there is a risk of damage to the gel and therefore the detectors are periodically recompressed. When high pressure ( 5.5 bars) is applied the bubbles will condense to liquid and the gel will return to the original shape and structure.

The high fluorine content of the active liquid makes PICASSO sensitive to spin dependent interactions [7], which are less well constrained than the spin independent interaction for WIMPs. While the cross section for spin independent interaction is proportional to the square of the mass number, the cross section for spin dependent interaction is instead proportional to an enhancement 


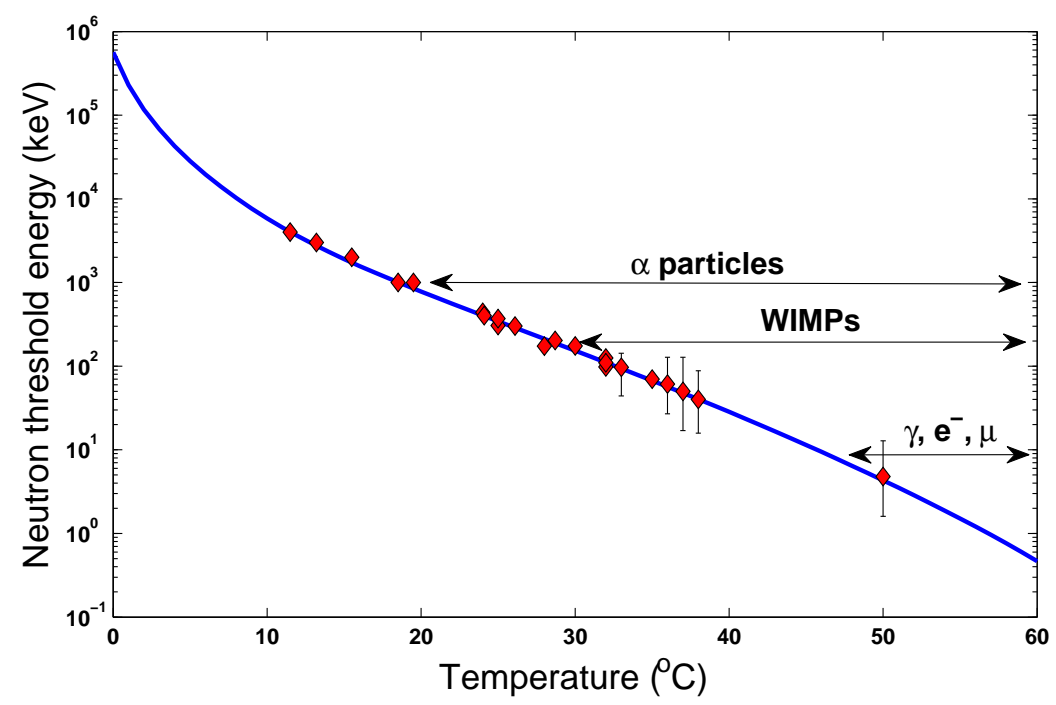

Figure 1: Neutron threshold energy as a function of temperature, at constant (atmospheric) pressure. The arrows indicate the temperature regions where PICASSO is sensitive to different types of particles.

factor depending on the nuclear structure. This enhancement factor is large for ${ }^{19} \mathrm{~F}$, which makes it a very favourable target material [8].

Other dark matter searches using superheated liquids are SIMPLE [1] and COUPP [2].

The present generation of the PICASSO modules are the largest bubble detectors ever constructed, with a volume of 4.5 litres. The gel with the fluorocarbon droplets is contained inside an acrylic vessel and covered with a layer of mineral oil. The container is sealed with a stainless steel lid, connected with a pipe to a hydraulic pressure system. Each module has nine piezoelectric sensors, allowing for the reconstruction of the location of the events in the detector.

For temperature control, four detectors modules are installed together inside an insulated aluminum box with heaters on the top and bottom plate. The whole detector contains eight of these temperature control boxes.

PICASSO is operated in SNOLAB, 2070 meters underground in the Creighton mine, Sudbury, Canada. The first detector modules from the current phase started taking data at the end of 2006, and the full installation of 32 detector modules is now nearly complete. The active mass of the full setup will be about 2.6 kilograms and in the current phase we will take $700 \mathrm{~kg}$ days of data.

Future plans involve background reduction of about two orders of magnitude, and a new detector generation with increased active mass.

\section{Analysis}

The analysis is based on understanding the backgrounds to look for an excess of events in the signal region.

The first step of the analysis is to filter the pulses from the piezoelectric sensors to remove noise events not related to bubble expansion. The useful waveforms contain a characteristic range 
of frequencies within a certain time window of the signal, and therefore the most important part of the filter is a selection on a Fourier transform of the signal. There are also cleaning steps to remove specific types of noise that sometimes will pass the frequency selection.

The event rate after the filter is plotted as a function of temperature, to get the response curve.

While it's not possible to separate the signal from the background on an event by event basis in the current analysis, the shape of the curve can be fitted with the known response to alphas and gammas. The WIMP signal should become visible as a contribution starting at higher temperatures than the alphas background, since the threshold for these events is at a lower energy corresponding to higher degree of superheat. If the fit agrees well with the data points and we see no excess of events starting at higher energies than gammas but lower than alphas, then we can calculate a limit on the cross section of the WIMPs for a range of masses. In case of detection, the threshold energy would give us some information about the particle properties.

Earlier published results (for a WIMP mass of $29 \mathrm{GeV} / \mathrm{c}^{2}$, a limit on the spin-dependent cross section on protons of $\sigma_{p}=1.31 \mathrm{pb}$ and on neutrons of $\sigma_{n}=21.5 \mathrm{pb}$ at $90 \%$ C.L.) [5] were based on a previous detector version with smaller modules. A new analysis of the data accumulated with the first of the installed modules in the current detector phase is on its way, and a publication with an updated limit is planned for this year.

\section{The recoil discrimination effect}

We recently discovered an unexpected difference between alphas and neutron induced nuclear recoils. When comparing the amplitude of the signals from alpha background data with data from calibration with a neutron source, the events fall in distinctly different classes (see figure 2).

The reason that this discrimination effect has not been seen earlier is that previous generations of PICASSO detectors had smaller droplets. Recall that the heat spike theory by Seitz [9] tells us that at least a critical energy $E_{C}$ has to be deposited within a critical length $L_{C}$ to induce a phase transition. A nucleus recoiling after a neutron interaction has a range shorter than $L_{C}$ and can only induce one proto-bubble. An alpha particle on the other hand typically has a range of several critical lengths, and a large droplet (100-200 $\mu \mathrm{m}$ ) can contain the whole track (about $34 \mu \mathrm{m}$ ).

Going from lower to higher temperatures, the alpha Bragg peak will first give signals at $21^{\circ} \mathrm{C}$. At higher temperatures the probability increases to get nucleation at more points along the track. If the phase transition starts at several nucleation centres it will progress faster, and this will result in a higher amplitude of the pressure wave. In this way the piezoelectric transducer signal contains some information about the early stage of the expansion.

The observed effect can be used to discriminate between alpha events and nuclear recoils in the analysis, and thereby reduce the background. Efforts are going on to optimize the gain of the sensor signals to get the most out of this discrimination.

\section{References}

[1] T. A. Girard et al.: "SIMPLE dark matter search results", Phys. Lett. B621 233, 2005

[2] P. Cooper, these proceedings, 2008 

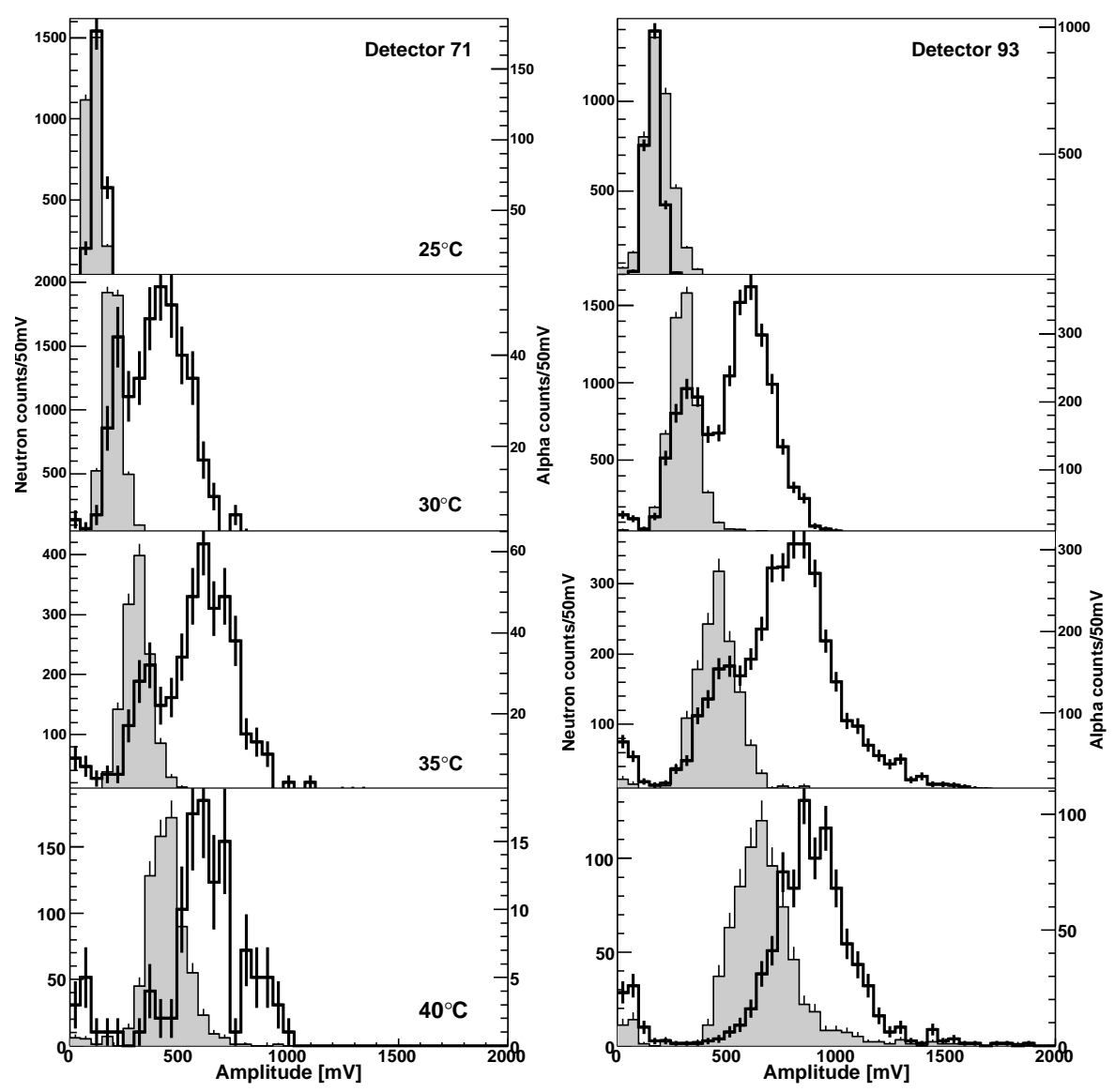

Figure 2: Alpha discrimination effect for two detector modules, at four different temperatures. The shaded histograms shows the pulse amplitudes from neutron dominated calibration events. In white are the same distributions from alpha dominated background data. The neutron background flux at the detectors is very low, but alpha contamination is limiting the sensitivity. The figure is taken from [4].

[3] M. Barnabe-Heider et al.: "Response of superheated droplet detectors of the PICASSO dark matter search experiment", Nucl.Instrum.Meth. A555 184, 2005

[4] F. Aubin et al.: "Discrimination of nuclear recoils from alpha particles with superheated liquids", New Journal of Physics 10, 2008

[5] M. Barnabe-Heider et al.: "Improved spin dependent limits from the PICASSO dark matter search experiment”,Phys.Lett.B624 186, 2005

[6] H. Ing et al.: "Bubble Detectors-A Maturing Technology", Radiat. Meas. 27 1:1, 1997

[7] J. Ellis and R.A. Flores: "Elastic supersymmetric relic-nucleus scattering revisited", Phys. Lett. B263 259,1991

[8] D.R. Tovey et al.: "A new model-independent method for extracting spin-dependent cross section limits from dark matter searches", Phys. Lett. B488 17, 2000

[9] F. Seitz: "On the Theory of the Bubble Chamber", Phys. Fluids 1 2, 1958 